\title{
An alternative polysaccharide uptake mechanism of marine bacteria
}

\author{
Greta Reintjes ${ }^{1}$, Carol Arnosti ${ }^{2}$, Bernhard M Fuchs ${ }^{1}$ and Rudolf Amann ${ }^{1}$ \\ ${ }^{1}$ Department of Molecular Ecology, Max Planck Institute for Marine Microbiology, Bremen, Germany and \\ ${ }^{2}$ Department of Marine Sciences, University of North Carolina-Chapel Hill, Chapel Hill, NC, USA
}

\begin{abstract}
Heterotrophic microbial communities process much of the carbon fixed by phytoplankton in the ocean, thus having a critical role in the global carbon cycle. A major fraction of the phytoplanktonderived substrates are high-molecular-weight (HMW) polysaccharides. For bacterial uptake, these substrates must initially be hydrolysed to smaller sizes by extracellular enzymes. We investigated polysaccharide hydrolysis by microbial communities during a transect of the Atlantic Ocean, and serendipitously discovered-using super-resolution structured illumination microscopy-that up to $26 \%$ of total cells showed uptake of fluorescently labelled polysaccharides (FLA-PS). Fluorescence in situ hybridisation identified these organisms as members of the bacterial phyla Bacteroidetes and Planctomycetes and the gammaproteobacterial genus Catenovulum. Simultaneous membrane staining with nile red indicated that the FLA-PS labelling occurred in the cell but not in the cytoplasm. The dynamics of FLA-PS staining was further investigated in pure culture experiments using Gramella forsetii, a marine member of Bacteroidetes. The staining patterns observed in environmental samples and pure culture tests are consistent with a 'selfish' uptake mechanisms of larger oligosaccharides ( $>600 \mathrm{Da})$, as demonstrated for gut Bacteroidetes. Ecologically, this alternative polysaccharide uptake mechanism secures substantial quantities of substrate in the periplasmic space, where further processing can occur without diffusive loss. Such a mechanism challenges the paradigm that hydrolysis of HMW substrates inevitably yields low-molecular-weight fragments that are available to the surrounding community and demonstrates the importance of an alternative mechanism of polysaccharide uptake in marine bacteria.
\end{abstract}

The ISME Journal (2017) 11, 1640-1650; doi:10.1038/ismej.2017.26; published online 21 March 2017

\section{Introduction}

Marine microbial communities are responsible for processing an estimated half of the organic carbon annually produced in the ocean (Azam and Malfatti, 2007). It is generally assumed that the highmolecular-weight (HMW) fraction of this organic matter is hydrolysed initially by extracellular enzymes to sizes <600 Da (Weiss et al., 1991) for transport into the cell (Arnosti, 2011). The conditions under which the production of an extracellular enzyme might be energetically beneficial, the extent to which diffusive loss of hydrolysed products limits the utility of extracellular enzyme production and scenarios under which non-enzyme-producing

Correspondence: C Arnosti, Department of Marine Sciences, University of North Carolina-Chapel Hill, Chapel Hill, NC 275993300, USA.

E-mail: arnosti@email.unc.edu

or R Amann, Department of Molecular Ecology, Max Planck Institute for Marine Microbiology, Celsiusstrasse 1, Bremen D-28359, Germany.

E-mail: ramann@mpi-bremen.de

Received 30 September 2016; revised 6 December 2016; accepted 22 January 2017; published online 21 March 2017 organisms may benefit from the activities of enzyme producers have been considered in recent models (for example, Vetter et al., 1998; Allison, 2005; Traving et al., 2015). These models assume that hydrolysis occurs in the extracellular environment and that hydrolysis products therefore are available to the wider microbial community. Models consequently typically express substrate availability in terms of monomer production and transport. Field measurements have likewise measured carbohydrate metabolism by microbial communities in the ocean via production and uptake of monosaccharides (for example, Rich et al., 1996). With few exceptions, moreover, investigations of carbohydrate dynamics have focussed primarily on enzymatic hydrolysis of glucose-containing substrate proxies (MUF- $\alpha$ - and $\beta$-glucose; Zaccone et al., 2012; Kellogg and Deming, 2014); glucose dynamics have also been used as a representation of polysaccharide metabolism in general (Christian and Karl, 1995; Piontek et al., 2014).

Different marine heterotrophs, however, specialise in uptake of low-molecular-weight and HMW substrates (for example, Cottrell and Kirchman, 2000; Elifantz et al., 2005, 2007). Natural microbial 
communities in surface ocean waters also exhibit substrate preferences. Differences in the spectrum of polysaccharides hydrolysed (Arnosti et al., 2005, 2012) and a latitudinal gradient in enzyme activities (Arnosti et al., 2011) that parallels large-scale patterns in microbial biogeography have been demonstrated (for example, Baldwin et al., 2005; Fuhrman et al., 2008; Wietz et al., 2010). The importance in marine systems of an alternative substrate uptake mechanism, known for gut bacteria (Bacteroidetes), has been suggested by metagenomic data from coastal ocean waters (Teeling et al., 2012, 2016) and oceanic provinces in the North Atlantic (Gómez-Pereira et al., 2012). With this mechanism, polysaccharides are bound to the outer membrane, partially hydrolysed, and transported as larger oligosaccharides ( $>600 \mathrm{Da}$ ) into the periplasm using TonB-dependent outer membrane receptors/transporters (Cho and Salyers, 2001), a mechanism homologous to the starch utilisation system (sus-like) (D'Elia and Salyers, 1996). Direct evidence of the manner in which individual bacteria in the ocean take up HMW polysaccharides, however, is still lacking.

To examine the links between activities and communities across broad spatial scales, we incubated natural microbial communities of five distinct oceanic provinces-Northern Temperate, Northern Gyre, Equatorial, Southern Gyre and Southern Temperate (Longhurst et al., 1995)_of the Atlantic Ocean (Supplementary Figure S1) with specific fluorescently labelled polysaccharides (FLA-PS). These polysaccharides, laminarin, xylan and chondroitin sulphate, were selected because they are present in large quantities in the ocean and/or enzymes that hydrolyse these polysaccharides are widely distributed among marine bacteria. For example, the production of laminarin, an energy storage product of diatoms, has been estimated at 5-15 billion metric tons annually (Alderkamp et al., 2007). Xylan is a major component of red and green algae (Lahaye et al., 1993; Usov, 2011) and thus widely present in the ocean, and chondroitin sulphate, which is commercially derived from shark cartilage, is rapidly and readily hydrolysed across broad ranges of ocean waters (Arnosti, 2011) and by a diverse array of marine bacterial isolates (Wegner et al., 2013; Xing et al., 2015). These polysaccharides also differ in chemical composition: laminarin is a glucose polysaccharide, xylan a polymer of xylose (a pentose rather than hexose sugar), and chondroitin sulphate is a sulphated polymer of $\mathrm{N}$-acetylgalactosamine and glucuronic acid. These substrates thus provide the opportunity to probe the activities of a wider range of enzymes (Arnosti, 2003).

We subsampled the incubations at sea and serendipitously observed, using epifluorescence microscopy, that up to $26 \%$ of the individual bacterial cells bound the FLA-PS. Pursuing these observations, we combined FLA-PS staining with single-cell identification by fluorescence in situ hybridisation (FISH) (Amann et al., 1995) and super-resolution light microscopy to visualise the uptake of FLA-PS by individual bacterial cells in natural communities in surface ocean waters.

\section{Materials and methods}

\section{Sampling and substrate incubations}

Seawater samples were collected aboard the RRV James Cook during the Atlantic Meridional Transect 22 cruise from Southampton, UK, to Punta Arenas, Chile, from 10 October to 24 November 2012. In five different oceanic provinces (Supplementary Figure S1) at solar noon, triplicate 20 litre seawater samples were collected from $20 \mathrm{~m}$ depth using a Niskin rosette with an attached Sea Bird CTD (Sea Bird Electronics Inc., Bellevue, WA, USA). From each triplicate, subsamples of $500 \mathrm{ml}$ were added to sterile glass bottles and incubated with one of the three FLA-PS, laminarin, chondroitin sulphate and xylan (nine bottles in total), for a total of 12-18 days. In addition, a treatment control, consisting of $500 \mathrm{ml}$ seawater in a sterile glass bottle without a FLA-PS, as well as killed controls, consisting of $50 \mathrm{ml}$ autoclaved seawater with one of the three FLA-PS, were incubated under the same conditions. All bottles were incubated at room temperature (RT) in the dark and sampled at regular time points (typically at 30 min, 1, 3, 6, 12 and 18 days). At each time point, samples for FISH analysis, measurement of extracellular enzyme activities and DNA analysis were collected. For FISH, $20 \mathrm{ml}$ of water was filtered through a $47 \mathrm{~mm}(0.2 \mu \mathrm{m}$ pore size $)$ polycarbonate filter, applying a gentle vacuum of $<200$ mbar. After drying, the filters were stored at $-20^{\circ} \mathrm{C}$ until further analysis. For DNA analysis, $10 \mathrm{ml}$ was filtered through a $0.2 \mu \mathrm{m}$ pore size polycarbonate filter using a Whatman 420200 Swin-Lok reusable filter holder (Sigma-Aldrich Chemie GmbH, Munich, Germany).

\section{FLA-PS synthesis and measurement of extracellular enzymatic activity}

Three polysaccharides (laminarin, xylan, chondroitin sulphate) obtained from Sigma-Aldrich (Munich, Germany) were fluorescently labelled with fluorescein amine (Sigma-Aldrich; isomer II) as described in Arnosti (2003). The FLA-PS solutions are free of monosaccharides or oligosaccharides, due to the fact that they are repeatedly injected onto standardised gel permeation chromatography systems as part of the labelling procedure; any low-molecular-weight carbohydrates are thereby removed during purification. Average-molecular weights of fluorescently labelled laminarin, xylan and chondroitin sulphate are 6000, 9000 and >50000 daltons, respectively. A single polysaccharide was added at a concentration of $1.75 \mu \mathrm{mol}$ monomer-equivalent to each $500 \mathrm{ml}$ water sample; each polysaccharide was incubated in triplicate, plus one killed control, as described above. 
Substrate staining, FISH and epifluorescence microscopy

For all the time points, the cells were filtered as described and counter-stained with 4',6-diamidino2-phenylindole (DAPI) and nile red and subsequently mounted using a Citifluor/VectaShield (4:1) mounting solution. Substrate-stained cells were visualised and enumerated using a fully automated microscope imaging system, described in detail by Bennke et al. (2016), on a Zeiss AxioImager.Z2 microscope stand (Carl Zeiss MicroImaging GmbH, Göttingen, Germany) with a cooled charged-coupleddevice (CCD) camera (AxioCam MRm; Carl Zeiss) and a Colibri LED light source (Carl Zeiss) with three light-emitting diodes (UV-emitting LED, $365 \pm 4.5 \mathrm{~nm}$ for DAPI; blue emitting LED, $470 \pm 14 \mathrm{~nm}$ for FLA-PS 488; red-emitting LED, $590 \pm 17.5 \mathrm{~nm}$ for the tyramide Alexa 594, FISH), combined with the HE-62 multifilter module (Carl Zeiss). This module consists of a triple emission filter TBP $425( \pm 25), 527( \pm 27)$, LP 615, including a triple beam splitter of TFT $395 / 495 / 610$. All automatic cell counts were validated using manual cell counting. Briefly automated cell counting was carried out by initially acquiring images (using a $63 \times$ magnification and 1.4 numerical aperture oil emersion plan apochromatic objective (Carl Zeiss)), at selected wavelengths (DAPI, FLA-PS, FISH), of a previously defined set of coordinates consisting of a minimum of 46 fields of view on each sample filter (Bennke et al., 2016). Subsequently, the images were imported into the ACMETOOL2.0 (http://www.technobiology.ch/ index.php?id = acmetool) image analysis software. From the images, cells were deemed 'substrate stained' if they showed a positive signal in both the DAPI and FLA-PS (488) images. Additionally, these signals had to have a minimum overlap of $30 \%$, a minimum area of 17 or 30 pixel $\left(0.17-0.3 \mu \mathrm{m}^{2}\right)$ (DAPI signal and FLA-PS signal, respectively) and a minimum signal background ratio of 1 or 2.5 (DAPI and FLA-PS signals, respectively) (Bennke et al. 2016).

FISH was carried out with slight alterations of the protocol by Manz et al. (1992). The hybridisation buffer contained $900 \mathrm{~mm}$ NaCl, $20 \mathrm{~mm}$ Tris-HCl $(\mathrm{pH}$ 7.5), $0.02 \%$ sodium dodecyl sulphate, $10 \%$ dextran sulphate (wt/vol) and 1\% (wt/vol) blocking reagent (Boehringer; Mannheim, Germany) with a formamide concentration optimised for individual probes (Supplementary Table S1). All hybridisations were carried out at $46^{\circ} \mathrm{C}$ in a humidity chamber for $3 \mathrm{~h}$, with a subsequent wash in a buffer containing 14-900 mm NaCl (dependent on formamide concentration in the hybridisation buffer), $20 \mathrm{~mm}$ Tris/HCl (pH 8), $5 \mathrm{~mm}$ EDTA (pH 8) and $0.01 \%$ sodium dodecyl sulphate at $48^{\circ} \mathrm{C}$. For super-resolution structured illumination microscopy (SR-SIM), the cells were initially scraped from the filter using a sterile scalpel and heat fixed to coverslips at $46^{\circ} \mathrm{C}$. After heat fixation, FISH was carried out as described above.
Super-resolution structured illumination microscopy Substrate incubation samples were visualised on a Zeiss ELYRA PS.1 (Carl Zeiss) using 561, 488 and $405 \mathrm{~nm}$ lasers and BP 573-613, BP 502-538 and BP 420-480+LP 750 optical filters. $Z$-stack images were taken with a Plan-Apochromat $63 \times / 1.4$ Oil objective and processed with the software ZEN2011 (Carl Zeiss). SR-SIM images are taken by exciting the sample using non-uniform wide-field illumination. The laser light passes through an optical grating, generating a striped-shaped sinusoidal interference pattern. This pattern then combines with the sample information originating from structures below the diffraction limit to generate moire fringes. The image is detected by a CCD camera and contains high spatial frequency sample information shifted to a lower spatial frequency band that is transmitted through the objective. Mathematical reconstructions from raw image slices then allow for a reconstruction of a high-resolution image with doubled resolution in the $x y$ plane (Schermelleh et al., 2010). Intensity line profiles of individual cells were carried out using the ZEN black software (Carl Zeiss).

\section{Medium preparation}

HaHa medium was prepared as described in detail by Hahnke and Harder (2013) and Hahnke et al. (2015). The basic HaHa medium was supplemented with $0.2 \mu \mathrm{m}$ sterile filtered carbon sources (glucose, cellobiose, yeast extract, peptone, casamino acids (Hahnke and Harder, 2013), laminarin (Sigma-Aldrich) and FLA-laminarin) to make different carbon source media (Supplementary Table S2).

\section{Pure culture FLA-PS incubations}

FLA-PS incubations were performed using a pure culture of Gramella forsetii strain KT0803 (DSM 17595), a marine member of Bacteroidetes. All growth experiments were carried out in biological duplicates. For all incubations and sampling time points (see below), cell growth and FLA-substrate uptake was analysed by fixing $1 \mathrm{ml}$ of culture using $2 \%$ sterile filtered formaldehyde for $1 \mathrm{~h}$ at RT. Subsequently, the sample was filtered through a $25 \mathrm{~mm}$ polycarbonate filter $(0.2 \mu \mathrm{m}$ pore size), applying a gentle vacuum of $<200 \mathrm{mbar}$, and the cells were visualised using microscopy (see 'Substrate staining, FISH and epifluorescence microscopy' section above). Cell fluorescence due to FLA-substrate uptake was quantified using an Accuri C6 flow cytometer (BD Accuri Cytometers, Ann Arbor, MI, USA).

$G$. forsetii was grown in $\mathrm{HaHa}$ high carbon medium (Supplementary Table S2) until it reached the stationary phase $(48 \mathrm{~h})$ and a cell count of $10^{7}$ cells $\mathrm{ml}^{-1}$. Subsequently, G. forsetii was inoculated $(1: 10)$ into HaHa minimal medium and grown for $48 \mathrm{~h}$; this was repeated twice to starve the culture and mimic a minimal carbon environment. To track 
the uptake of FLA-laminarin by G. forsetii, in a low carbon environment, this starved culture was inoculated (1:10) into HaHa FLA-laminarin $35 \mu \mathrm{M}$ medium and sampled every $3 \mathrm{~h}$.
To allow for the upregulation of gene expression and production of enzymes capable of laminarin uptake (induction), the starved G. forsetii culture was inoculated into $\mathrm{HaHa}$ laminarin medium
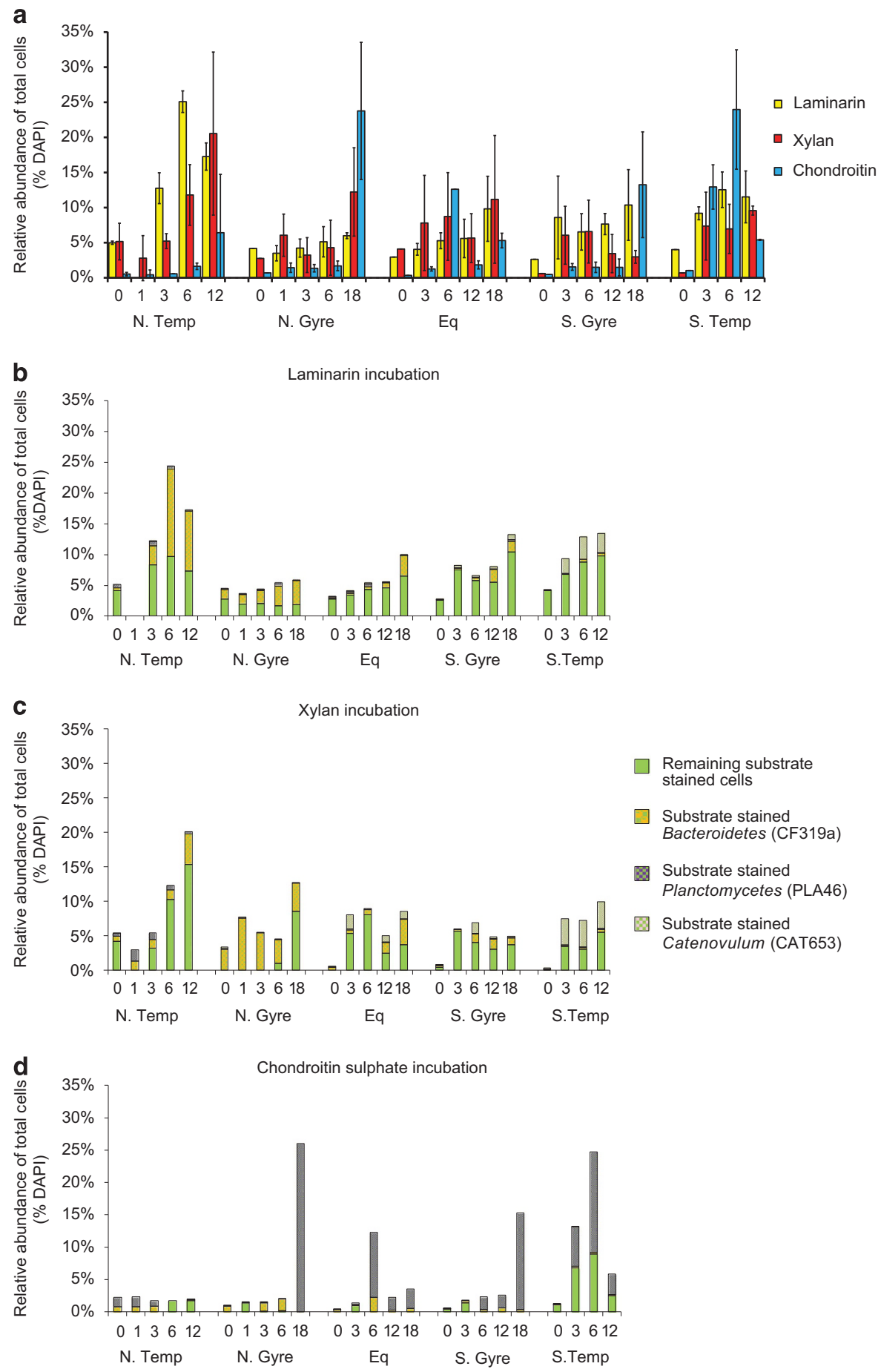

Figure 1 Relative abundance of substrate-stained cells and FISH identification of substrate-stained cells in incubations of seawater from five stations of different provinces of the Atlantic Ocean (see Supplementary Figure S1 for station locations). (a) relative abundance of cells stained by laminarin (yellow), xylan (red) and chondroitin sulphate (blue). Incubation time is indicated by ' $T$ ' ( $0-18$ days). $T 0$ refers to samples taken approximately 30 min after addition of the FLA-PS. (b) Relative abundance of laminarin-stained cells and fraction stained by FISH probes for Bacteroidetes (CF319a), Planctomycetes (PLA46) and Catenovulum (CAT653). (c) Relative abundance of xylan-stained cells and fraction stained by FISH probes for Bacteroidetes (CF319a), Planctomycetes (PLA46) and Catenovulum (CAT653). (d) Relative abundance of chondroitin-stained cells and fraction stained by FISH probes for Bacteroidetes (CF319a), Planctomycetes (PLA46) and Catenovulum (CAT653). 
for $48 \mathrm{~h}\left(1 \times 10^{6}\right.$ cells $\left.\mathrm{ml}^{-1}\right)$. This was also carried out to test whether the uptake of FLA-laminarin increased after induction of the cells, mimicking an environment where laminarin is already available. Induced $G$. forsetii was inoculated (1:10) in both HaHa FLA-laminarin $35 \mu \mathrm{M}$ medium and HaHa FLA-laminarin $3.5 \mu \mathrm{M}$ medium. Substrate staining was tracked by sampling at 5, 20, 40, 60, 80 and 100 min.

To further analyse the FLA-substrate uptake by the cells and to determine whether the FLA tag can be excreted, after $100 \mathrm{~min}$ incubation in $\mathrm{HaHa}$ FLA-laminarin $35 \mu \mathrm{m}$ medium, the substrate-stained cells were inoculated (1:10) in HaHa laminarin. The decrease in FLA signal was analysed by sampling at 20, 40,60, 80, $100 \mathrm{~min}$ and 1 day after inoculation.

All growth experiments without FLA-laminarin were sampled regularly to check for autofluorescence or other sources of fluorescence that could be mistaken for substrate signals. Additionally, to ensure that there was no unspecific binding of FLA-laminarin to cells, cells grown in $\mathrm{HaHa}$ high carbon medium and HaHa laminarin medium were fixed using $2 \%$ sterile filtered formaldehyde for $1 \mathrm{~h}$ at RT and subsequently incubated with $35 \mu \mathrm{M}$ FLAlaminarin for $4 \mathrm{~h}$. The cells were then filtered through a $25 \mathrm{~mm}(0.2 \mu \mathrm{m}$ pore size) polycarbonate filter, applying a gentle vacuum of $<200 \mathrm{mbar}$ and visualised using microscopy. There was no unspecific binding of substrate to fixed cells.

\section{Flow cytometry and fluorescence quantification}

Cell fluorescence due to FLA-substrate uptake was quantified in all $G$. forsetii growth cultures using an Accuri C6 flow cytometer (BD Accuri Cytometers). Initially, the cells were fixed in $37 \%$ sterile filtered formaldehyde (final concentration $2 \%$ ) for $1 \mathrm{~h}$ at RT. The 8- and 6-peak validation bead suspensions (Spherotech, Lake Forest, IL, USA) were used as internal references. The cells were analysed under laser excitation at $488 \mathrm{~nm}$ from a blue-green diode laser and the green fluorescence was collected in the FL1 channel $(530 \pm 30 \mathrm{~nm})$. An electronic threshold of 10000 FSC-H was set to reduce background noise. All samples were analysed at the same flow rate (slow) and a total of 20000 events per sample were acquired. Bacteria were detected from the signature plot of SSC-H vs green fluorescence (FL1-H). The FCM output was analysed using the BD Accuri software. Cells were assumed to give a positive signal if their associated mean fluorescence intensity was greater than the FL1-H of a culture not incubated in FLA-laminarin. As $G$. forsetii can form aggregates over time, a subset of data was defined using gates that represented single cells. For these gates, comparative fluorescence intensity was carried out by comparison of the mean fluorescence intensity to that of non-FLA-laminarin-stained cells.
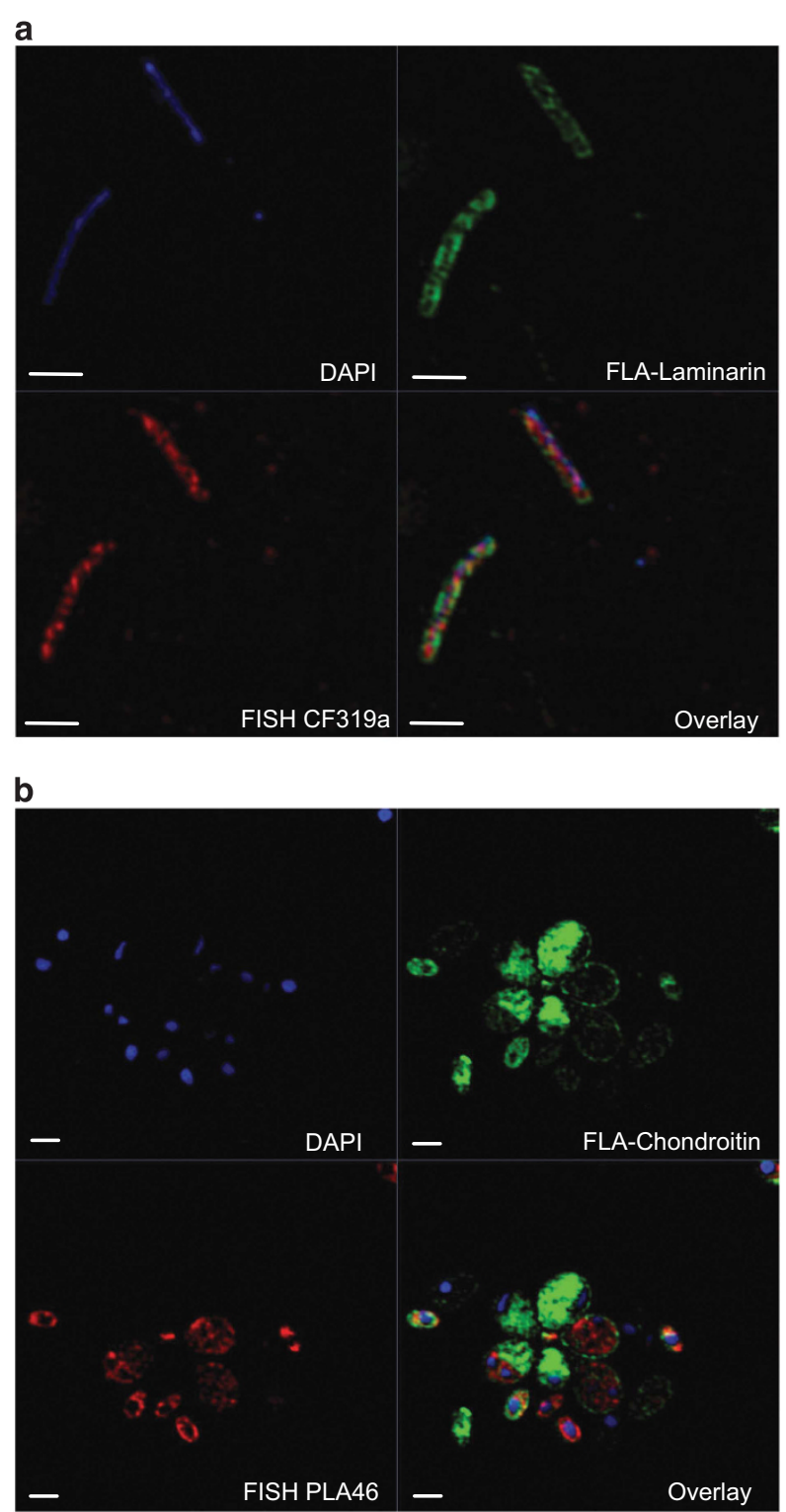

Figure 2 SR-SIM of cells stained by (a) DAPI (blue), FLAlaminarin (green) and Bacteroidetes-specific FISH probe (CF319a, red); (b) DAPI (blue), FLA-chondroitin sulphate (green) and Planctomycetes-specific FISH probe (PLA46, red). Scale bar $=1 \mu \mathrm{m}$.

\section{Results and discussion}

We incubated sea water from five distinct oceanic provinces with three FLA-PS and observed by epifluorescence microscopy that a considerable fraction of the bacterial community-up to $26 \%$ of total cells (Figure 1a)-bound detectable amounts of substrate (Figure 2,Supplementary Figure S2). Fluorescent staining was seen in all incubations but varied considerably with substrate and station (Figure 1a). The highest overall abundance and most rapid staining of cells was usually seen in the laminarin incubations. For example, in the Northern Temperate province $5 \%$ of cells show staining after just 
$30 \min (T 0)$ and the abundance increased to $25 \%$ after 6 days. Xylan staining of cells was also seen quickly (5\% at T0) in the Northern Temperate, Northern Gyre and Equatorial stations but not in the Southern Gyre or Southern Temperate stations. The highest abundance of xylan-stained cells was in the Northern Temperate station after 12 days (20\%). In the chondroitin sulphate incubations, the increase of substrate-stained cells occurred more slowly. High abundances of $22 \pm 3 \%$ were nonetheless observed at later time points (T18) in the gyre stations. At the Southern Temperate station, a high percentage of cells were stained already at T6. Many different cell morphologies were observed among substratestained cells-coccoid, rod shaped and ovoid; the rate of staining of the different cell types varied (Figure 2,Supplementary Figure S2). A diverse range of bacteria thus were binding FLA-PS.

Examination of individual cells using SR-SIM, which enables visualisation of prokaryotic cell compartments, showed that the initial association of cells with substrates occurred in the cell periphery within $30 \mathrm{~min}$ (Supplementary Figure S4a). Such rapid direct staining was surprising, as polysaccharide additions were moderate (са. $21 \mu \mathrm{M}$ C) and the average number of fluorophores per molecule of polysaccharide (the labelling density) was low (between 0.5 and 1.3 per polysaccharide molecule; Arnosti (2003). Currently, it is not possible to measure the absolute number of fluorophores taken up over time by individual cells in an environmental sample. However, a comparison of the FLA-PS signal and the FISH signal resulting from a $4 \times$ labelled rRNA targets oligonucleotide of the same cell (Figure 2) suggests that thousands of FLA-PS molecules have been bound by individual cells.

Rapid staining was also observed in pure cultures of G. forsetii incubated with FLA-laminarin (Supplementary Figure S3 and Supplementary Table S3). When G. forsetii cells grown on a minimal carbon medium with no polysaccharide were inoculated into a medium containing FLA-laminarin (35 $\mu \mathrm{M}$ monomer $\mathrm{l}^{-1}$ ), it took up to $12 \mathrm{~h}$ for substrate-specific staining to be observed. However, when $G$. forsetii was grown on a laminarin medium and subsequently inoculated into FLA-laminarin $\left(3.5 \mu \mathrm{M}\right.$ monomer $\left.\mathrm{l}^{-1}\right)$ containing media, staining could be seen within minutes (Supplementary Figure S3 and Supplementary Table S3). This result not only shows the high affinity of induced $G$. forsetii towards laminarin but also demonstrates that a fraction of the environmental bacteria were likely induced or specialised for the immediate uptake of polysaccharides, as seen by staining at T0 (Figure 1a and Supplementary Figure S4).

Live G. forsetii lost much of the substrate signal from FLA-laminarin within $24 \mathrm{~h}$ (Supplementary Table S3). Specifically, when G. forsetii was transferred from HaHa FLA-laminarin medium into $\mathrm{HaHa}$ laminarin medium, cells continued growing and simultaneously lost the FLA-laminarin signal over time. The slow removal of FLA from the cell indicates that the substrate was not unspecifically bound to the cell surface but instead taken up into the cell. Moreover, the loss of signal in G. forsetii over time cannot be solely related to dilution through cell division, as the signal decreased more rapidly than average doubling times of $G$. forsetii.

In environmental samples, the overall abundance of substrate-stained cells increased with time in all incubations $\left(R^{2}=0.0823, \quad P\right.$-value $\left.=0.0153\right)$ (Figure 1a), despite the fact that (with a single exception) the absolute cellular abundances within the incubations did not increase significantly (Supplementary Figure S5). Although the abundance of stained cells increased, this relationship varied by station and substrate. At the Equatorial station (xylan and chondroitin sulphate incubations) and the Southern Gyre station (laminarin and xylan incubations), moreover, there was a decrease in the abundance of substrate-stained cells between day 3 and day 6. No substrate staining was detected in heat-killed or formaldehyde-fixed cell controls, indicating that staining was due to biological activity (Supplementary Figure S6).

The ability to use polysaccharides has been confirmed for many marine bacterial phyla, including Bacteroidetes, Planctomycetes, Verrucomicrobia and Proteobacteria (Martinez-Garcia et al., 2012; Teeling et al., 2012; Kabisch et al., 2014; Lage and Bondoso, 2014; Wietz et al., 2015). Based on the literature (Schattenhoffer et al., 2009; Teeling et al., 2012; Lage and Bondoso, 2014; Wietz et al., 2015) and cell morphologies, a selection of group-specific FISH probes (Supplementary Table S1) was used to identify and enumerate specific bacterial group abundances at each time point and station. The combination of FISH with substrate staining allowed for the identification of organisms directly taking up a specific substrate (Figures 2a and b). Using this probe set (CF319a, PLA46 and CAT653 targeting the Bacteroidetes, Planctomycetes and Catenovulum, respectively), an average of $48 \% \pm 49 \%$ (median of $55 \%$ ) of the substrate-stained cells could be identified (Figures 1b-d). Future analysis using 16S rRNA sequencing is currently being pursued to supplement this probe set in future and further increase the fraction of identified cells.

The FISH counts of the substrate incubations of the Atlantic Ocean showed an increase in abundance of the selected clades. For example, FISH counts of the laminarin incubation at the Northern Temperate station showed a nearly fivefold increase in Bacteroidetes abundance over 6 days (from $7.2 \times 10^{4}$ to $3.3 \times 10^{5}$ cells ml ${ }^{-1}$ at day 6), with $80 \%$ of these cells showing substrate-specific staining (Figure $1 \mathrm{~b}$ and Supplementary Figure S9). In the other regions, Bacteroidetes did not increase as strongly in abundance, possibly due to lower initial cell numbers, but the percentage of substrate stained Bacteroidetes increased from $5 \%$ to $72 \pm 28 \%$ in $12-18$ days. 
Planctomycetes cellular abundances likewise increased, particularly in the chondroitin sulphate incubation of the Southern Temperate station, from
$2.3 \times 10^{3}$ to $2.5 \times 10^{5}$ cell $\mathrm{ml}^{-1}$ over 6 days; $84 \%$ of these cells showed substrate staining (Figure $1 \mathrm{~d}$ and Supplementary Figure S9). The substrate-stained
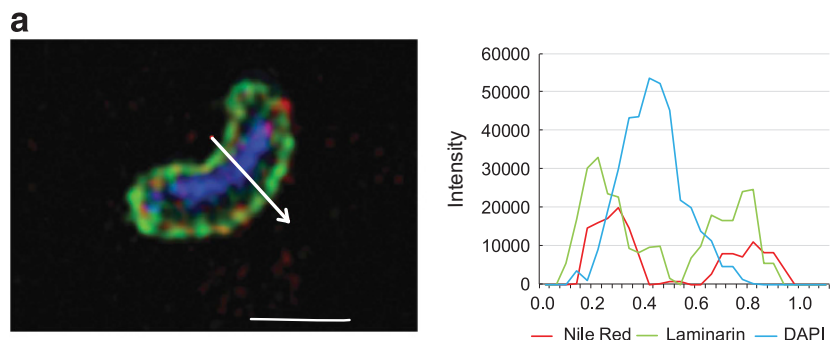

b

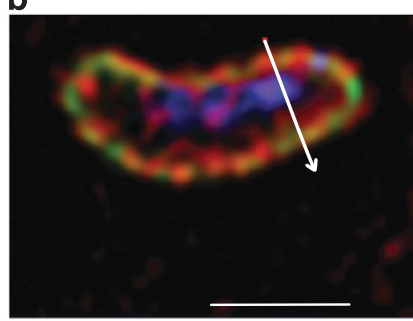

c

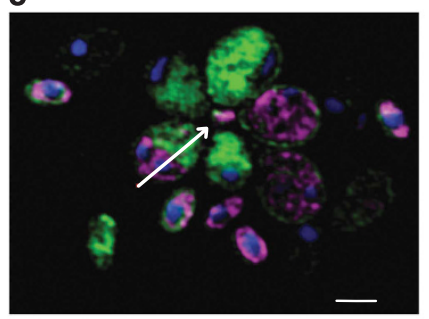

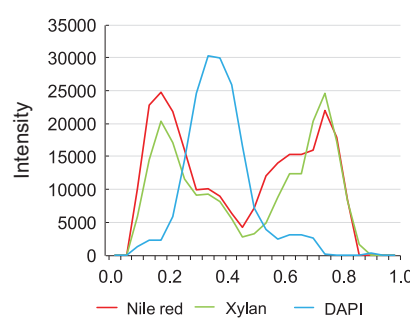

- Nile red - Xylan - DAPI

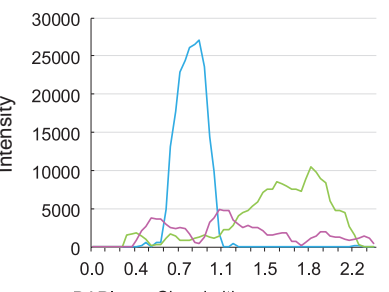

— DAPI - Chondroitin — FISH (PLA46)

d

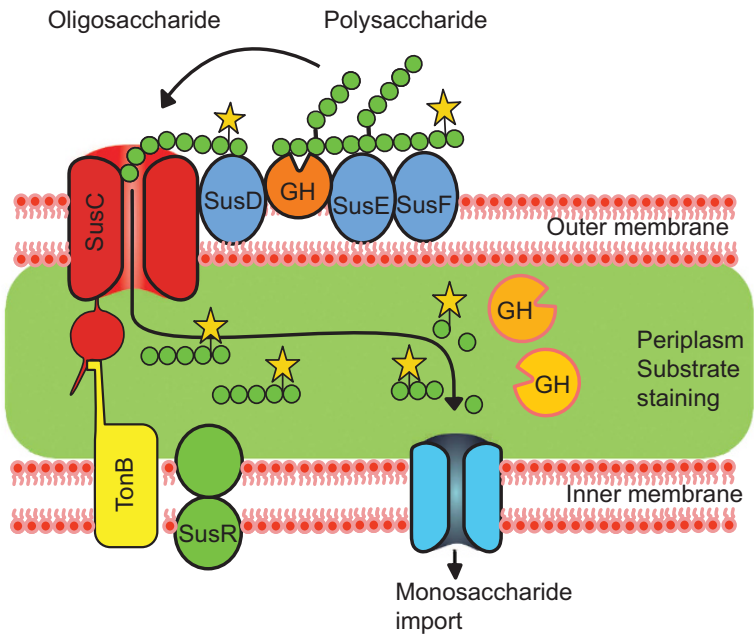

Figure 3 SR-SIM images showing Sus-like uptake of FLA-PS and fluorescence intensity line profiles localising substrate-specific staining after 6 days of incubation. (a and b) Cells stained with FLA-PS (green), nile red (red; membrane) and DAPI (blue; DNA). White arrows indicate sections along which the fluorescence intensity line profiles were recorded. Scale bars $=1 \mu \mathrm{m}$. Corresponding profiles indicating co-localisation of substrate and membrane are shown on the right. (a) Bacteroidetes cell from the Northern Temperate station stained with FLA-laminarin. (b) Bacteroidetes cell from the Southern Temperate station stained with FLA-xylan. (c) Planctomycetes cells from the Southern Temperate station stained with FLA-chondroitin. Cells were identified using the FISH probe PLA46 (magenta), which labels the riboplasm; the substrate staining is in the paryphoplasm. (d) Conceptual model of sus-like bacterial uptake of FLA-PS into the periplasm via TonB-dependent outer membrane transporters, causing halo-like staining in periplasm (green). The large oligosaccharides are further hydrolysed within the periplasm to monosaccharides, disaccharides or trisaccharides, which are subsequently transported into the cytoplasm. Modified after Koropatkin et al. (2012). 'GH' represents glycoside hydrolases. 
cells in the chondroitin sulphate incubations of other regions exhibited distinctive Planctomycetes-like cell morphologies but were only partially identified as Planctomycetes with FISH probe PLA46, perhaps due to difficulty of permeabilising Planctomycetes cells (Pizzetti et al., 2011). In addition to the Bacteroidetes and Planctomycetes, the gammaproteobacterial genus Catenovulum also showed substrate-specific staining. Catenovulum increased in abundance in the laminarin and xylan incubations at the Equatorial, Southern Gyre and Southern Temperate stations and constituted $1-4 \%$ of the total stained cells.

Substrate-stained cells predominantly exhibited a halo-like staining that was restricted to the cell periphery (Figures 3a and b,Supplementary Figure S8). Fluorescence intensity line profiles of individual cells in combination with nile red membrane counterstaining showed a co-localisation of the substrate signal with the cell membranes (Figures 3a and b). However, in cells $<0.5 \mu \mathrm{m}$ in width, for example, some Bacteroidetes, the halo-like staining could not, due to limits of optical resolution, definitively be shown to be within the periplasmic space between the membranes. In contrast, for the larger cells of Planctomycetes uptake of FLAchondroitin sulphate across the outer membrane could be seen (Figure 3c and Supplementary Figure S7). SR-SIM revealed that FLA-chondroitin sulphate had been transported into the paryphoplasm but not into the riboplasm (Figure 3c and Supplementary Figure S7). Similar to the periplasm of Gram-negative bacteria, the paryphoplasm is a cell compartment located between an inner and an outer membrane that does not contain ribosomes (Fuerst and Sagulenko, 2011).

Genomic analyses of marine strains from Bacteroidetes and Planctomycetes have shown that they have the potential to express carbohydrate transporters for the uptake of large oligosaccharides through the outer membrane (Fuerst and Sagulenko, 2011; Teeling et al., 2012; Paparoditis et al., 2014). Intestinal Bacteroidetes in particular are known for sus-like polysaccharide utilisation loci (Koropatkin et al., 2012). When expressed, the proteins encoded in these loci are principally located in the outer membrane and the periplasm. They sequentially bind and hydrolyse polysaccharides, transporting large oligosaccharides into the periplasm, where further degradation occurs in a protected space (Figure 3d; Koropatkin et al., 2012). Our observations of the staining patterns of marine Bacteroidetes cells are consistent with this mode of substrate processing. Previous research on G. forsetii has shown that, in laminarin-amended cultures, the expression of proteins required for its binding, transport and utilisation of laminarin is induced (Kabisch et al., 2014). Here we show that induced G. forsetii cells are stained with FLA-laminarin in minutes (Supplementary Figure S3), whereas cells that were not induced required hours before staining can be detected. Up to $5 \%$ of open ocean bacteria are readily stained with FLA-laminarin, showing detectable staining after just $30 \mathrm{~min}(\mathrm{T0})$, which indicates that for these bacteria no induction is required: they are primed for rapid uptake of laminarin into the periplasm. (Supplementary Figure S4a).

Based on our microscopic examination of Bacteroidetes from the surface ocean, data from pure cultures of $G$. forsetii and genomic information (Koropatkin et al., 2012; Teeling et al., 2012; Kabisch et al., 2014), we hypothesise that the substrate uptake we observed is homologous to the sus-like mechanism of gut Bacteroidetes. This mechanism has recently been referred to as 'selfish' by Cuskin et al. (2015) owing to the fact that, after uptake of large oligosaccharides, further degradation occurs in the protected periplasmic space. Selfish substrate uptake confers a distinct ecological benefit by minimising formation of monosaccharides, disaccharides and trisaccharides in the external environment and avoiding diffusive loss of enzymes (Figure 3d; Cuskin et al., 2015). The uptake of chondroitin sulphate by Planctomycetes likely occurs via an analogous but unknown mechanism.

As demonstrated by the substantial fraction of the natural microbial community that was stained by just three distinct substrates (Figure 1a), this substrate utilisation strategy is important not only in the anaerobic and organic-carbon rich environment of the human gut (Cuskin et al., 2015) but also in the oxic, dilute and comparatively organic-carbon-poor surface waters of the Atlantic Ocean. Although these experiments were carried out in bottle incubations that may well have led to changes in the microbial community from its initial composition, the organisms growing later in the incubations must ultimately have originated from the seawater sample we collected. The activity seen later in the incubations thus may not reflect the exact dynamics that occur in the ocean, but nonetheless highlight the selfish uptake capabilities that these microorganisms possess. Moreover, the observation that FLA-PS were bound by organisms in both the early (initial $30 \mathrm{~min}$ ) and late (up to 18 days) phases of the incubations suggest that this strategy of substrate acquisition competes well with alternative strategies of substrate utilisation in complex microbial communities. These observations imply that a re-evaluation of models of bacterial substrate utilisation in natural environments will be necessary. Current models typically encompass two classes of organisms, those that produce enzymes that release low-molecular-weight substrates to the environment and those that use the hydrolysis products but do not produce enzymes themselves (for example,Allison, 2005; Kaiser et al., 2015; Traving et al., 2015). These two-player models will need to be expanded to consider organisms that have evolved mechanisms to minimise substrate sharing. 


\section{Conclusions}

Measurements and models of the manner in which the most abundant products of photosynthesis, polysaccharides, are channelled into the microbial food chain need to account for the varying ecological strategies of heterotrophic marine bacteria. Most field measurements of enzyme activities rely on substrate proxies containing monomers (Zaccone et al., 2012; Kellogg and Deming, 2014); carbohydrate uptake measurements in ocean waters likewise are made most frequently with monosaccharides (Rich et al., 1996). These measurements do not account for bacteria that quickly capture and process HMW polysaccharides. The speed and extent of selfish substrate uptake by phylogenetically distinct bacteria at five widely spaced stations in the Atlantic Ocean demonstrate that this is an important mechanism of carbon utilisation that previously has been overlooked. Future models as well as measurements will need to account for this mode of substrate acquisition as part of microbially driven carbon cycling in the ocean.

The FLA-PS staining method, in combination with FISH, allows for direct identification of polysaccharide-degrading bacteria in environmental samples. Based on this new method, future studies can specifically measure the types and quantities of phytoplankton-produced polysaccharides that are processed by this mechanism, as well as explore other locations and conditions under which selfish substrate utilisation may predominate.

\section{Conflict of Interest}

The authors declare no conflict of interest.

\section{Acknowledgements}

We thank the captain and crew of the RRV James Cook, as well as the principle scientist Glen Tarran (Plymouth Marine Laboratories), for assistance at sea. We also thank Jörg Wulf, David Probandt, Marion Stagars, Andreas Ellrott and Sherif Ghobrial for technical assistance and Ian Head, Cindy Lee and Andreas Teske for helpful discussions. This work was supported by the Max Planck Society and by the U.S. National Science Foundation (OCE-1332881 to CA). CA was additionally supported in part by a fellowship from the Hanse Institute for Advanced Study (Delmenhorst, Germany). This study is a contribution to the international IMBER project and was also supported by the UK Natural Environment Research Council National Capability funding to Plymouth Marine Laboratory and the National Oceanography Centre, Southampton. This is contribution number 281 of the AMT program.

\section{Author contributions}

Experimental design: RA, CA, and GR; sampling: GR and RA; FISH and cell counting, automated microscopy and super-resolution microscopy: GR; fluorescent labelling of polysaccharides: CA; probe design: $\mathrm{BF}$. The manuscript was written primarily by GR, CA and RA with contributions from BF. GR prepared the figures.

\section{References}

Alderkamp A-C, Van Rijssel M, Bolhuis H. (2007). Characterization of marine bacteria and the activity of their enzyme systems involved in degradation of the algal storage glucan laminarin. FEMS Microbiol Ecol 59: $108-117$.

Allison SD. (2005). Cheaters, diffusion and nutrients constrain decomposition by microbial enzymes in spatially structured environments. Ecol Lett 8: 626-635.

Amann RI, Ludwig W, Schleifer KH. (1995). Phylogenetic identification and in situ detection of individual microbial cells without cultivation. Microbiol Rev 59: 143-169.

Arnosti C. (2003). Fluorescent derivatization of polysaccharides and carbohydrate-containing biopolymers for measurement of enzyme activities in complex media. J Chromatogr B 793: 181-191.

Arnosti C, Durkin S, Jeffrey WH. (2005). Patterns of extracellular enzyme activities among pelagic marine microbial communities: implications for cycling of dissolved organic carbon. Aquat Microb Ecol 38: 135-145.

Arnosti C. (2011). Microbial extracellular enzymes and the marine carbon cycle. Annu Rev Mar Sci 3: 401-425.

Arnosti C, Steen AD, Ziervogel K, Ghobrial S, Jeffrey WH. (2011). Latitudinal gradients in degradation of marine dissolved organic carbon. PLoS One 6: e28900.

Arnosti C, Fuchs BM, Amann R, Passow U. (2012). Contrasting extracellular enzyme activities of particle-associated bacteria from distinct provinces of the North Atlantic Ocean. Front Microbiol 3: 425.

Azam F, Malfatti F. (2007). Microbial structuring of marine ecosystems. Nat Rev Microbiol 5: 782-791.

Baldwin AJ, Moss JA, Pakulski JD, Catala P, Joux F, Jeffrey WH. (2005). Microbial diversity in a Pacific Ocean transect from the Arctic to Antarctic circles. Aquat Microb Ecol 41: 91-102.

Bennke CM, Reintjes G, Schattenhofer M, Ellrott A, Wulf J, Zeder $\mathrm{M}$ et al. (2016). Modification of a highthroughput automatic microbial cell enumeration system for ship board analyses. Appl Environ Microbiol 82: 3289-3296.

Cho KH, Salyers AA. (2001). Biochemical analysis of interactions between outer membrane proteins that contribute to starch utilization by Bacteroides thetaiotaomicron. J Bacteriol 183: 7224-7230.

Christian JR, Karl DM. (1995). Bacterial ectoenzymes in marine waters: activity ratios and temperature responses in three oceanographic provinces. Limnol Oceanogr 40: 1042-1049.

Cottrell MT, Kirchman DL. (2000). Natural assemblages of marine Proteobacteria and members of the CytophagaFlavobacter cluster consuming low- and highmolecular-weight dissolved organic matter. Appl Environ Microbiol 66: 1692-1697. 
Cuskin F, Lowe EC, Temple MJ, Zhu Y, Cameron EA, Pudlo NA et al. (2015). Human gut Bacteroidetes can utilize yeast mannan through a selfish mechanism. Nature 517: 165-169.

D’Elia JN, Salyers AA. (1996). Effect of regulatory protein levels on utilization of starch by Bacteroides thetaiotaomicron. J Bacteriol 178: 7180-7186.

Elifantz H, Malmstrom RR, Cottrell MT, Kirchman DL. (2005). Assimilation of polysaccharides and glucose by major bacterial groups in the Delaware estuary. Appl Environ Microbiol 71: 7799-7805.

Elifantz H, Dittel AI, Cottrell MT, Kirchman DL. (2007). Dissolved organic matter assimilation by heterotrophic bacterial groups in the western Arctic Ocean. Aquat Microb Ecol 50: 39-49.

Fuerst JA, Sagulenko E. (2011). Beyond the bacterium: planctomycetes challenge our concepts of microbial structure and function. Nat Rev Microbiol 9: 403-413.

Fuhrman JA, Steele JA, Hewson I, Schwalbach MS, Brown MV, Green JL et al. (2008). A latitudinal diversity gradient in planktonic marine bacteria. Proc Natl Acad SCi 105: 7774-7778.

Gómez-Pereira PR, Schüler M, Fuchs BM, Bennke C, Teeling H, Waldmann J et al. (2012). Genomic content of uncultured Bacteroidetes from contrasting oceanic provinces in the North Atlantic Ocean. Environ Microbiol 14: 52-66.

Hahnke RL, Harder J. (2013). Phylogenetic diversity of Flavobacteria isolated from the North Sea on solid media. Syst Appl Microbiol 36: 497-504.

Hahnke RL, Bennke CM, Fuchs BM, Mann AJ, Rhiel E, Teeling $\mathrm{H}$ et al. (2015). Dilution cultivation of marine heterotrophic bacteria abundant after a spring phytoplankton bloom in the North Sea. Environ Microbiol 17: 3515-3526.

Kabisch A, Otto A, König S, Becher D, Albrecht D, Schüler $\mathrm{M}$ et al. (2014). Functional characterization of polysaccharide utilization loci in the marine Bacteroidetes 'Gramella forsetii' KT0803. ISME J 8: 1492-1502.

Kaiser C, Franklin O, Richter A, Dieckmann U. (2015). Social dynamics within decomposer communities lead to nitrogen retention and organic matter build-up in soils. Nat Commun 6: 8960.

Kellogg CTE, Deming JW. (2014). Particle-associated extracellular enzyme activity and bacterial community composition across the Canadian Arctic Ocean. FEMS Microbiol Ecol 89: 360-375.

Koropatkin NM, Cameron EA, Martens EC. (2012). How glycan metabolism shapes the human gut microbiota. Nat Rev Microbiol 10: 323-335.

Lage OM, Bondoso J. (2014). Planctomycetes and macroalgae, a striking association. Front Microbiol 5: 133.

Lahaye M, Michel C, Barry JL. (1993). Chemical, physicochemical and in-vitro fermentation characteristics of dietary fibres from Palmaria palmata (L.) Kuntze. Food Chem 47: 29-36.

Longhurst A, Sathyendranath S, Platt T, Caverhill C. (1995). An estimate of global primary production in the ocean from satellite radiometer data. J Plankton Res 17: 1245-1271.

Manz W, Amann R, Ludwig W, Wagner M, Schleifer K-H. (1992). Phylogenetic oligodeoxynucleotide probes for the major subclasses of proteobacteria: problems and solutions. Syst Appl Microbiol 15: 593-600.
Martinez-Garcia M, Brazel DM, Swan BK, Arnosti C, Chain PSG, Reitenga KG et al. (2012). Capturing single cell genomes of active polysaccharide degraders: an unexpected contribution of Verrucomicrobia. PLoS One 7: e35314.

Paparoditis P, Västermark Å, Le AJ, Fuerst JA, Saier MH Jr. (2014). Bioinformatic analyses of integral membrane transport proteins encoded within the genome of the planctomycetes species, Rhodopirellula baltica. Biochim Biophys Acta 1838: 193-215.

Piontek J, Sperling M, Nöthig E-M, Engel A. (2014). Regulation of bacterioplankton activity in Fram Strait (Arctic Ocean) during early summer: the role of organic matter supply and temperature. J Mar Syst 132: 83-94.

Pizzetti I, Fuchs BM, Gerdts G, Wichels A, Wiltshire KH, Amann R. (2011). Temporal variability of coastal planctomycetes clades at kabeltonne station, North Sea. Appl Environ Microbiol 77: 5009-5017.

Rich JH, Ducklow HW, Kirchman DL. (1996). Concentrations and uptake of neutral monosaccharides along $14^{\circ}$ W in the equatorial Pacific: contribution of glucose to heterotrophic bacterial activity and the DOM flux. Limnol Oceanogr 41: 595-604.

Schattenhofer M, Fuchs BM, Amann R, Zubkov MV, Tarran GA, Pernthaler J. (2009). Latitudinal distribution of prokaryotic picoplankton populations in the Atlantic Ocean. Environ Microbiol 11: 2078-2093.

Schermelleh L, Heintzmann R, Leonhardt H. (2010). A guide to super-resolution fluorescence microscopy. J Cell Biol 190: 165-175.

Teeling H, Fuchs BM, Becher D, Klockow C, Gardebrecht A, Bennke CM et al. (2012). Substrate-controlled succession of marine bacterioplankton populations induced by a phytoplankton bloom. Science 336: $608-611$.

Teeling H, Fuchs BM, Bennke CM, Krüger K, Chafee M, Kappelmann L et al. (2016). Recurring patterns in bacterioplankton dynamics during coastal spring algae blooms. eLife 5: e11888.

Traving SJ, Thygesen UH, Riemann L, Stedmon CA. (2015). A model of extracellular enzymes in free-living microbes: which strategy pays off? Appl Environ Microbiol 81: 7385-7393.

Usov AI. (2011). Chapter 4-Polysaccharides of the red algaeIn:Horton D(ed). Advances in Carbohydrate Chemistry and Biochemistry. Academic Press, pp 115-217.

Vetter YA, Deming JW, Jumars PA, Krieger-Brockett BB. (1998). A predictive model of bacterial foraging by means of freely released extracellular enzymes. Microb Ecol 36: 75-92.

Wegner C-E, Richter-Heitmann T, Klindworth A, Klockow C, Richter M, Achstetter T et al. (2013). Expression of sulfatases in Rhodopirellula baltica and the diversity of sulfatases in the genus Rhodopirellula. Mar Genomics 9: $51-61$.

Weiss M, Abele U, Weckesser J, Welte W, Schiltz E, Schulz G. (1991). Molecular architecture and electrostatic properties of a bacterial porin. Science 254: 1627-1630.

Wietz M, Gram L, Jørgensen B, Schramm A. (2010). Latitudinal patterns in the abundance of major marine bacterioplankton groups. Aquat Microb Ecol 61: 179.

Wietz M, Wemheuer B, Simon H, Giebel H-A, Seibt MA, Daniel R et al. (2015). Bacterial community dynamics during polysaccharide degradation at contrasting sites 
in the Southern and Atlantic Oceans. Environ Microbiol 17: 3822-3831.

Xing P, Hahnke RL, Unfried F, Markert S, Huang S, Barbeyron $\mathrm{T}$ et al. (2015). Niches of two polysaccharide-degrading Polaribacter isolates from the North Sea during a spring diatom bloom. ISME $J$ 9: $1410-1422$.

Zaccone R, Boldrin A, Caruso G, La Ferla R, Maimone G, Santinelli C et al. (2012). Enzymatic activities and prokaryotic abundance in relation to organic matter along a West-East Mediterranean Transect (TRANSMED Cruise). Microb Ecol 64: $54-66$.
This work is licensed under a Creative Commons Attribution 4.0 International License. The images or other third party material in this article are included in the article's Creative Commons license, unless indicated otherwise in the credit line; if the material is not included under the Creative Commons license, users will need to obtain permission from the license holder to reproduce the material. To view a copy of this license, visit http:// creativecommons.org/licenses/by/4.0/

(C) The Author(s) 2017

Supplementary Information accompanies this paper on The ISME Journal website (http://www.nature.com/ismej) 